\title{
Spherical disharmonics in the Earth sciences and the spatial solution: Ridges, hotspots, slabs, geochemistry and tomography correlations
}

\author{
Terrill W. Ray and Don L. Anderson \\ Seismological Laboratory, California Institute of Technology, Pasadena
}

\begin{abstract}
Alsstract. There is increasing use of statistical correlations between geophysical fields and between geochemical and geophysical fields in attempts to understand how the Earth works. Typically, such correlations have been based on spherical harmonic expansions. The expression of functions on the sphere as spherical harmonic series has many pitfalls, especially if the data are nonuniformly and/or sparsely sampled. Many of the difficulties involved in the use of spherical harmonic expansion techniques can be avoided through the use of spatial domain correlations, but this introduces other complications, such as the choice of a sampling latice. Additionally, many geophysical and geochemical fields fail to satisfy the assumptions of standard statistical significance tests. This is especially problematic when the data values to be correlated with a geophysical field were collected at sample locations which themselves correlate with that field. This paper examines many correlations which have been claimed in the past between geochemistry and mantle tomography and between hotspot, ridge, and slab locations and tomography using both spherical harmonic coefficient correlations and spatial domain correlations. No conclusively significant correlations are found between isotopic geochemistry and mantle tomography. The Crough and Jurdy (short) hotspot location list shows statistically significant correlation with lowermost mantle tomography for degree 2 of the spherical harmonic expansion, but there are no statistically significant correlations in the spatial case. The Vogt (long) hotspot location list does not correlate with tomography anywhere in the mantle using either technique. Both hotspot lists show a strong correlation between hotspot locations and geoid highs when spatially correlated, but no correlations are revealed by spherical harmonic techniques. Ridge locations do not show any statistically significant correlations with tomography, slab locations, or the geoid; the strongest correlation is with lowermost mantle tomography, which is probably spurious. The most striking correlations are between mantle tomography and post-Pangean subducted slabs. The integrated locations of slabs correlate strongly with fast areas near the transition zone and the core-mantle boundary and with slow regions from 1022-1284 km depth. This seems to be consistent with the "avalanching" downwellings which have been indicated by models of the mantle which include an endothermic phase transition at the $670-\mathrm{km}$ discontinuity, although this is not a unique interpretation. Taken as a whole, these results suggest that slabs and associated cold downwellings are the dominant feature of mantle convection. Hotspot locations are no better correlated with lower mantle tomography than are ridge locations.
\end{abstract}

\section{Introduction}

Statistical correlations between gcophysical fields and between geophysical and geochemical fields have seen significant use in the never-ending effort to learn how the Earth works. One of the most popular methods of correlating global gcophysical fields involves the use of spherical harmonic coefficients. When the function is known everywhere on the sphere, it is easy to compute the spherical harmonic coefficients using the standard integral formulation. With a function neither known on the entire sphere nor sampled on a fine enough lattice, we must use a different technique. Typically, a least squares fitting technique is used. The most straightforward way of doing this is to take the sampled points and directly fit the spherical harmonics to the data points [Cain et al., 1965]. Other possibilities include fitting to interpolated values [Leaton et al.,

Copyright 1994 by the American Geophysical Union.

Paper number 94JB00340.

0148-0227/94/94JB-00340\$05.00
1965], fitting to area polynomials which have been fitted to the data [Hurwitz et al., 1966], or fitting to a Gram-Schmidt orthogonalization fit [Fougere, 1965]. All of these fitting techniques share the common problem that the spherical harmonic coefficients computed by these methods are dependent on the number of terms included in the expansion.

\section{Correlation of Spherical Harmonic Coefficients}

Eckhardt [1984] discusses a technique for calculating the correlation coefficients between two sets of spherical harmonic coefficients and also for determining the significance levels of such correlations. These correlations are performed degree-bydegree, because the functions of spherical harmonic coefficients (the amplitudes of the spherical harmonics) tend to be highly skewed in the case of most geophysical fields [Eckhardt, 1984] and it is very problematic to evaluate the significance of correlations between highly skewed functions.

Although spherical harmonics are extremely powerful in dealing with functions defined on a sphere, they should not be 
used indiscriminately. When the function is everywhere known on the sphere, spherical harmonic expansion provides a compact and straightforward way of expressing the function. If the function is only known at discrete sample points the sample points must be spaced evenly and closely enough that they sample the highest degree in which there is significant power.

\section{Real-Space Correlations}

\section{Linear Correlation of Data}

The preceding discussion suggests that it might be appropriate to search for methods of correlating fields without stepping into the hazardous domain of spherical harmonics. The simplest and most straightforward technique would be to sample the functions defined on the sphere at $N$ discrete points (where the $N$ points are typically the points at which the more poorly sampled data set is known) and to use the resulting data pairs in well-known correlation algorithms such as simple linear correlations [Press et al., 1986].

\section{Correlating Locations}

It is often desirable to calculate the correlation between locations on the sphere and another spherical function, for example, hotspot locations and tomography. It is necessary to understand how the location of sampling points on the sphere may be influencing the correlation of the field being sampled.

While the function of sampling point locations is known over the entire sphere and may straightforwardly be expanded into spherical harmonics, it is inappropriate, because one does not wish to smooth this function. If the correlation of the sampled field is done spatially, instead of making use of spherical harmonics, expanding the data point locations is doubly inappropriate.

Take a function known on the entire sphere and sample it at each location where data in the other set, which is known only at discrete sample locations, is known. If the value of the function is negative, assign $x_{i}=-1$; if positive, assign $x_{i}=1$. A correlation coefficient can then be calculated using

$$
r=\frac{\sum_{i} x_{i} \sin ^{2}\left(\text { colat }_{i}\right)}{\sum_{i} \sin \left(\text { colat }_{i}\right) \sum_{i} \sin \left(\text { colat }_{i}\right)}
$$

where colat $_{i}$ is the colatitude of sample point $i$. This test is centered at

$$
\frac{p-q}{2}
$$

where $p$ is the proportion of the map covered by positive values and $q$ is the proportion of the map covered by negative areas. Significance testing may be performed using a binomial distribution:

$$
\delta(x)=\left(\begin{array}{l}
N \\
X
\end{array}\right) p^{X} q^{N-X}=\frac{N !}{X !(N-X) !} p^{X} q^{N-X}
$$

where $f(X X)$ is the probability of $X$ out of $N$ points randomly placed on a map landing on regions with positive values.

\section{Statistical Significances}

Statistical significance testing yields the probability that rejecting the null hypothesis is incorrect. The null hypothesis, in terms of correlation, is that the two data sets are uncorrelated.
The standard significance tests make further assumptions regarding the individual and joint distributions of the data sets, and these assumptions are fundamentally a part of the null hypothesis being tested. Even if the result of the statistical significance testing indicates that the chance of incorrectly rejecting the null hypothesis is extremely small, it may be simply that the assumptions on the data distributions are grossly incorrect but that the functions are still uncorrelated.

In a sense, standard significance testing assumes that the dice are not loaded. The generally used null hypothesis assumes that the functions are completely uncorrelated, and the binomial distribution test of the location correlations assumes that the probability of success $p$ is independent of previous trials. In the case of correlating oceanic ridge locations with seismic tomography, we know that the ridge segments form a line and that many positive points will lie next to each other in the tomography map. In the case of hotspots, any correlation between hotspot locations and tomography will influence the comelation of data collected at the hotspots (e.g., geochemistry, swell height, and flux) and tomography.

In the spatial correlations below, the following significance test was used, given that one function is known over the entire sphere. The set of data on the sphere at only selected points is rotated, keeping the points in the same positions relative to each other. The two functions are correlated with the second in the new position, and this process is repeated for a large number (2000) of random rotations. This yields a distribution of correlation coefficients which may be used to determine the significance of the calculated correlation coefficient. This tests for the probability of the given point pattern correlating with a randomly oriented map having the same structure as the globally known map. This test determines whether the point pattern has a tendency to produce a skewed distribution of correlation coefficients. If the correlation coefficient being tested tends to fall outside the region encompassing $95 \%$ of the correlation coefficients arising from the Monte Carlo test, the correlation is judged to be significant. This allows us to avoid identifying correlation coefficients which may arise from the fact that the point distribution and the map it is being correlated with tend to produce a skew distribution of correlation coefficients. The case for points placed uniformly and randomly on the sphere should be adequately encompassed through the use of the binomial distribution test mentioned previously.

All of the assumptions made in the null hypothesis, including the assumption that the two functions are uncorrelated, must be examined and verified or negated before a meaningful decision is made to accept or reject a statistically significant correlation. Further, if some of the assumptions being made in the null hypothesis are incorrect, a result which appears to be statistically insignificant may be significant. It should always be kept in mind that significance testing normally does not tell if an alternative hypothesis is correct; it can only indicate the probability that the null hypothesis is being incorrectly rejected.

\section{Correlations Involving Geochemical and Geophysical Fields}

Castillo [1988] claimed to find a correlation between the maxima of the Dupal [Hart, 1984] geochemical anomaly and slow regions of lower mantle tomography. This was a simple qualitative correlation using an integrated map of spherical harmonic degrees 2 and 3 of the entire lower mantle. It was argued that these correlations could help constrain lower mantle 
convection because of the isolation time which some geochemists feel would be needed to create the isotopic signature of the Dupal anomaly. Unfortunately, the qualitative nature of this correlation makes it nearly impossible to determine the significance of the correlation, and the use of an integrated lower mantle tomography map makes it impossible to judge where, if anywhere, in the vertical dimension of the lower mantle these correlations occur. A more accurate knowledge of where the correlations occur would be required to give any meaningful constraints on mantle convection.

Richards et al. [1988] expanded the global hotspot distribution into spherical harmonics and correlated the distribution with the geoid and mantle tomography for each spherical harmonic degree. They found statistically significant (>90\% confidence) correlations at $l=2$ between hotspots and both geoid and lower mantle tomography. Weaker correlations between hotspots and the geoid were found at $l=4$ and $l=6$. Richards and Engebretson [1992] correlated a spherical harmonic expansion of time-integrated slab positions with average lower mantle seismic velocity and found statistically significant (>90\%) correlation only for $l=2$ and $l=3$.

Kedar et al. (1994) expanded hotspot locations into a spherical harmonic representation and correlated degree by degree with the 11-layer Tanimoto [1990] tomography model. Statistically significant correlations were found in the deeper upper mantle (200-670 km depth) for $l=6$ and in the bottom half of the lower mantle $(1555-2891 \mathrm{~km})$ for $l=2$. The spherical harmonic expansion of the hotspot distribution was found to be dominated by $l=1$ and $l=2$ with another smaller peak at $l=6$, and these facts were used to argue that the correlations were not merely statistically significant but in fact scientifically significant as well.

Scrivner and Anderson [1992] expanded maps of the Pangea supercontinent and post-Pangeatic subduction (integrated slab) into spherical harmonics and calculated correlation coefficients between these maps and three seismic tomography models [Tanimoto, 1990; Su and Dziewonski, 1991; Inoue et al., 1990]. Pangea was found to have a statistically significant correlation for $l=1$ with the upper mantle for two of the three tomography models. Post-Pangeatic subduction had statistically significant correlations with the transition zone for $l=2$ in all of the tomography models, with the peak correlation occurring near $550 \mathrm{~km}$ depth. Importantly, in both cases, the statistically significant correlations occurred in the degree having the maximum power in the spherical harmonic expansion $(l=1$ for Pangea, $l=2$ for post-Pangeatic subduction). Correlation coefficients were also calculated between the hotspot distribution and integrated slab with statistically significant correlations occurring at $l=3$ and $l=5$ which both have relatively high power in the subduction expansion but low power in the hotspot expansion.

The Tanimoto [1990] tomography model is used in all of the following correlations. The subducted slab map of Scrivner and Anderson [1992] and the hydrostatic geoid from Gaposchkin [1974] will be used in order to see if any correlations exist between these maps and the geochemistry. We might expect correlations between geochemistry and slab, because subducting slab would represent a geochemical heterogeneity in the mantle, and we might expect a correlation with the geoid since the geoid shape is controlled by internal mass distribution and by the motion of material within the mantle.

The geochemical data used in this study are ocean island basalt (OIB) data given in Hart [1984], Zindler et al. [1982],
Gill [1984], Vidal et al. [1984], Roden et al. [1984], and Richardson et al. [1982]; and mid-ocean ridge basalt (MORB) data from Ito et al. [1987]. Figure 1 shows the locations of all of the data points, with the OIB points as open circles and the MORB points as closed circles. Hart [1984] expresses isotopic data as deviations from reference lines, and this method is followed in this paper. The reference levels for strontium and neodymium used in this study were taken as the median values of the respective isotopic ratios in the OIB data set. The reference level is very important if one wishes to use the integral technique to calculate the spherical harmonic coefficients, since the integral assumes that all points not assigned specific values are zero, but it is not as important when using least squares to evaluate the coefficients. The linear correlation techniques are not at all dependent on the reference levels.

A list of 47 hotspots used by Richards et al. [1988], Kedar et al. [1994], and Scrivner and Anderson [1992] which is based on the compilation of Morgan [1981] and Crough and Jurdy [1980] is used in this study and henceforth referred to as the "short" list. A list of 117 hotspots compiled by Vogt [1981] will be referred to as the "long" list. The post-Pangean subduction map of Scrivner and Anderson [1992] is used to correlate slab subduction with tomography (Figure 2). It is noted that this map is based on tracing the locations of trenches since the breakup of Pangea and may not fully consider the amount of slab subducted in each location. The ridge map is based on a $1^{\circ}$ by $1^{\circ}$ map where a value of 1 is assigned to the bin if it contains a ridge segment and a value of zero is assigned if it does not.

\section{Spherical Harmonic Correlations}

The spherical harmonic coefficients for each isotopic data set, hotspot location list, ridge location, and integrated slab location were calculated using a numerical impementation of the standard integral expression for spherical harmonic coefficients [Scrivner and Anderson, 1992; Kedar et al., 1994]. For the isotopic data, the value of the deviation from the reference line is assigned to each grid point, with zeros assigned to all points lacking data (zero indicates that the isotopic ratio does not deviate from the reference line). Figure 1 is a map of the spherical harmonic expansion for $\Delta 8 / 4$, where white regions are regions of positive deviations from the reference line and the shaded regions are regions of negative deviations from the reference line. Notice that the pattern resulting from this expansion does not resemble either the Hart [1984] structure of a band of high $\Delta 8 / 4$ values at about $30^{\circ} \mathrm{S}$ latitude nor the Castillo [1988] structure of unconnected highs in the Indian Ocean and the West Pacific Ocean. This does not mean that the raw data fail to support either of these models for the high $\Delta 8 / 4$ zone; it may simply mean that a spherical harmonic expansion from degree 0 through degree 6 fails to adequately describe the data. It is, however, an unbiased way to represent the data. The hotspot, ridge, and subducted slab locations can be defined for every point on the sphere, so there is no real barrier to expressing these data distributions in terms of spherical harmonics. However, a low order expansion may lead to undesirable smoothing of these functions.

Table 1 summarizes the significant ( $>90 \%$ confidence level) spherical harmonic correlations of geochemistry, both hotspot location lists, mid-oceanic ridge locations, and the integrated slab map of Scrivner and Anderson [1992] shown in Figure 2. Eckhardt [1984] gives definitions of confidence levels for spherical harmonic correlations. The spherical harmonic degree 


\section{D8/4 Expansion OIB \& MORB Data}

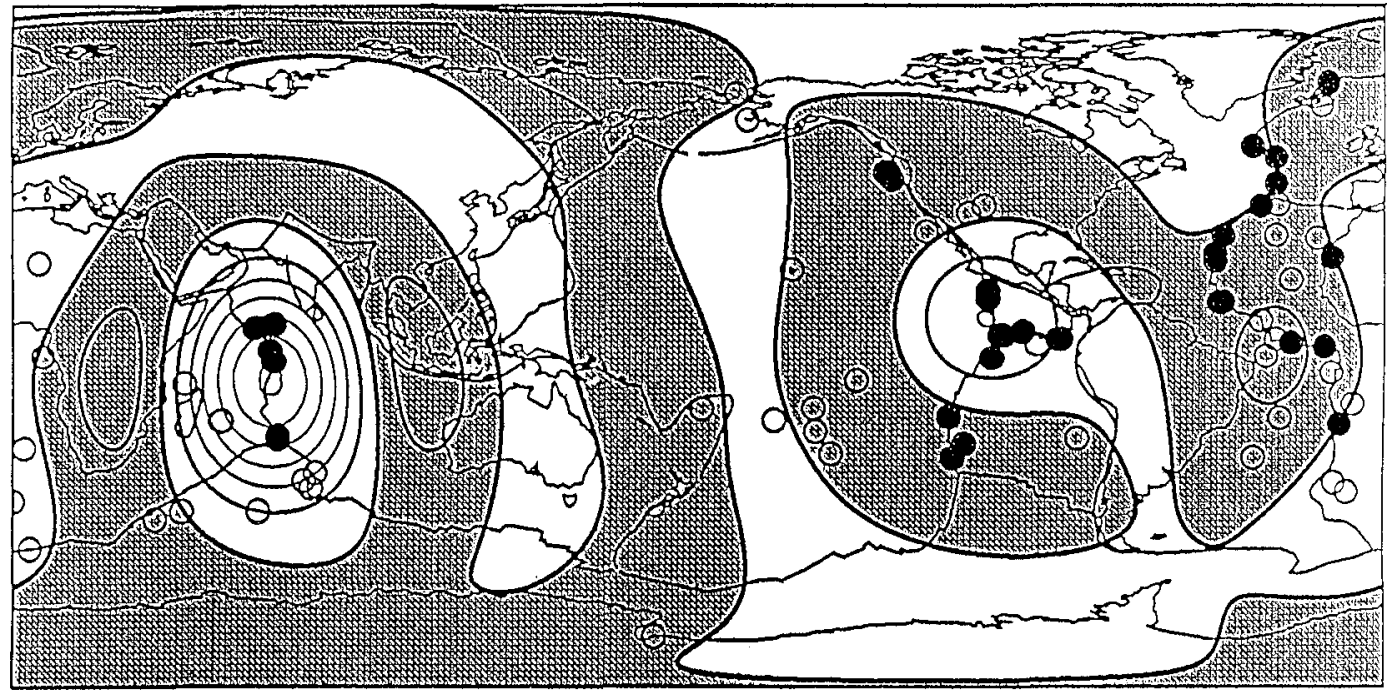

OIB - O

MORB -

Figure 1. Map of degrees 0-6 of the spherical harmonic expansion of the $\Delta 8 / 4$ isotopic data. MORB sample locations are shown by solid circles, and OIB sample locations are indicated by open circles. Shaded regions are regions of negative deviations from the isotopic reference line.

and the sign of the correlation are given in the first column of Table 1. The parameter being tested is given in the first row. The entries in the table are the Tanimoto layer designations ( $1=$ surface and $11=$ core-mantle boundary), slab (s), or geoid (g).

A notable feature not appearing in this table is the geoid which did not correlate significantly in any degree with any of the other parameters. Layer 5 occurs most often in this table, with 15 appearances (out of a possible 108), and layer 4 is the second most frequently occurring (12 appearances). Layers $1-6$ account for 57 of the significant correlations while layers 7-11 account for only 16 , with slab accounting for 10 . Considering only the geochemical correlations, layers $1-6$ account for 26 correlations, while layers 7-11 account for only 7, and slab accounts for 4. This distribution of significant correlations would seem to imply that the upper half of the mantle has the most influence on both geochemistry and the locations of features such as hotspots and ridges.

There are very few robust correlations. Most of the apparently significant correlations do not carry over to adjacent degrees, layers, or parameters. The notable exceptions are the strong negative degree 2 correlation between the short list of hotspot locations and layers $7-11$ of the Tanimoto tomography

\section{Post-Pangea Slab Locations: degree 1-8}

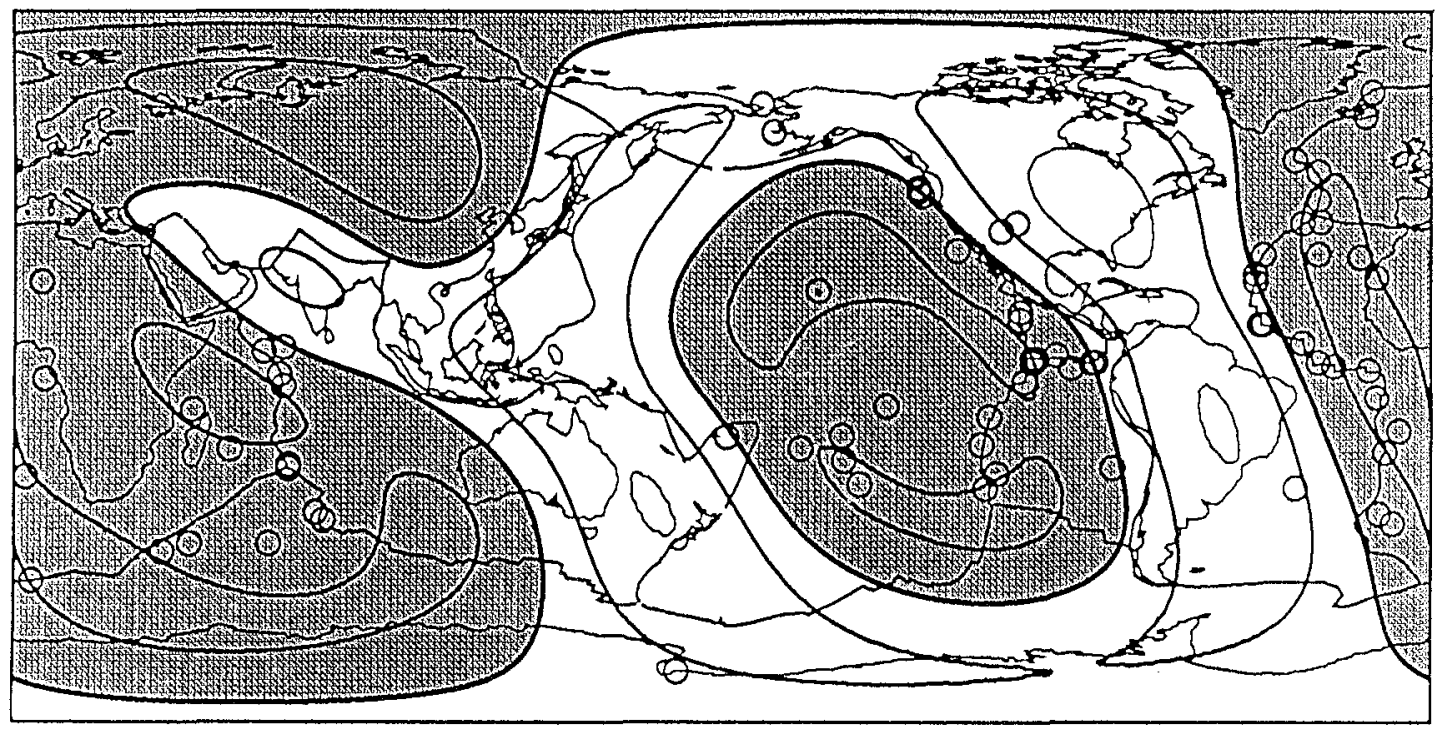

Figure 2. Plot of degree 1-8 expansion of post-Pangean subduction (subducted slab). White regions indicate the presence of subducted slab, and shaded regions indicate its absence. 
Table 1. Significant Spherical Harmonic Correlations

\begin{tabular}{cccccccccc}
\hline Degree \& & $\mathrm{Pb}$ & $\mathrm{Pb}$ & $\mathrm{Sr}$ & $\mathrm{Nd}$ & $\mathrm{Nd}$ & & & Ridge & Subducted \\
Sign of & $(\Delta 7 / 4)$ & $(\Delta 8 / 4)$ & $(\Delta \mathrm{Sr})$ & $(\Delta \mathrm{Nd})$ & Data & Hotspot Locations & Locations & Slab \\
& & & & & Locations & Short List & Long List & & Locations \\
\hline
\end{tabular}

$+6$

$+5$

$+4$

$+3$

$+2$

$+1$

$-1$

$-2$

$-3$

$-4$

$-5$

$-6$

$\begin{array}{cc}5 & 5 \\ & 6 \\ & 1 \\ & \\ \text { s } & \text { s } \\ 7 & \\ 5,6,9,10,11 & 10,11 \\ & 3,4,5 \\ 1 & 1 \\ 1 & \end{array}$

3,5

2,5

$1,4,5$

6

6,7

6

6

6

4

4

$7,8,9,10,11$

$5, \mathrm{~s}$

$4,5, \mathrm{~s}$

6

4

$2,3,5,11$

$1, \mathrm{~s}$

5

4

5

5

$\begin{array}{ccc} & 4 & 3 \\ & 2 & \\ & \mathrm{~s} & \\ 1,2 & 5,10,11 \\ 1, \mathrm{~s} & & \end{array}$

Column headings indicate the parameter being correlated. Table entries indicate what layers of tomography correlate (1, surface; 11, core-mantle boundary), where $g$ indicates geoid and $s$ indicates slab.. Notice that there are no statistically significant correlations with the geoid. Data is at the $>90 \%$ confidence level.

and the strong negative degree 6 correlation between the short list of hotspots and layers $2-4$ of the tomography, both of which were reported by Kedar et al. [1994]. Also robust are the positive degree 2 correlations between subducted slab and layers $2-4$ of the tomography and the positive degree 3 correlations beiween subducted slab and layers 4,6 , and 7 of the tomography, as reported by Scrivner and Anderson [1992]. Among the geochemical data, the most robust correlations are the negative degree 3 correlations of $\Delta 7 / 4$ with layers 9-11 of the tomography and the negative degree 3 correlations of $\Delta 8 / 4$ and layers $10-11$ of the tomography. These geochemical correlations are also apparent when the MORB data are excluded. The negative degree 4 correlations between $\Delta 8 / 4$ and layers 3-5 of the tomography may also be robust, but there is not a similar set of correlations for any other isotope. Taken in sum, these spherical harmonic correlations do not appear to give us even marginally consistent relationships between the geochemistry on the surface and mantle convection inferred from the tomography, regardless of the fact that there are many individually statistically significant correlation coefficients between the various isotopes and the mantle tomography. Furthermore, if we look back at Figure 1, we can see that the data points are not well distributed on the globe, which serves as a warning against the use of spherical harmonic expansions.

\section{Spatial Domain Correlations}

In this case we take the values in the isotopic data sets and average them in $5^{\circ} \times 5^{\circ}$ bins. For each bin having at least one data value we calculate the value of the spherical harmonic series for the tomography, geoid, or slab at that location. The resulting set of data pairs is then correlated using simple linear correlation.

Figure 3 shows the results of the spatial correlation for the geochemical data. The solid triangles connected with the solid line are plotted at the correlation coefficients for each layer, with the geoid and slab as labeled and the rest of the numbered layers corresponding to the Tanimoto [1990] tomography model layers.
The dashed line is the $95 \%$ confidence level from the standard confidence level test for linear correlation, and $95 \%$ of the correlation coefficients calculated using the Monte Carlo type test discussed previously fall within the unadomed solid lines, so these serve as $95 \%$ confidence level lines. The Monte Carlo test takes into account both the distribution of data points and the structure of each tomography map and the slab and geoid maps. The Monte Carlo test should also account for the distribution of the data values. For these reasons, the Monte Carlo test is probably more useful. Notice that no correlations lie outside the $95 \%$ confidence level lines from the Monte Carlo test.

Only a few geochemical correlations exceed the $95 \%$ confidence level for the standard test. These correlations tend to occur in the lowermost mantle layers and are of a negative sense for $\Delta 7 / 4$ and $\Delta \mathrm{Sr}$ and of a positive sense for $\Delta \mathrm{Nd}$. Also of interest are the layer 1 correlations of $\Delta \mathrm{Sr}$ and $\Delta \mathrm{Nd}$. The deep mantle correlations are perhaps slightly suggestive of enriched isotopic ratios being related to slow (presumably hot) regions of the lowermost mantle, but these correlations are not robust under the Monte Carlo test.

Now consider the ridge, hotspots, and slab locations and use the location correlation described by equation (1). Each of the $5^{\circ} \times 5^{\circ}$ bins which contain any hotspot, ridge, or slab (depending on which set of locations we wish to correlate) is assigned a value of 1 . Then the tomography, geoid, and slab spherical harmonic series are evaluated at those points, and a value of 1 is given to the bin if the resulting value is positive and a value of -1 if it is negative. Points not containing the discrete feature type are ignored.

The reason for ignoring the bins which do not contain the feature of interest is that in the case of hotspots and ridges, the number of bins not containing these elements far outweighs the number containing these elements, resulting in a correlation which reveals very little about how the locations of hotspots (or ridges) correlate with the tomography, geoid, or slab. As a case of this, consider the problem of correlating the Vogt list of 
$\triangle 7 / 4$ Correlations

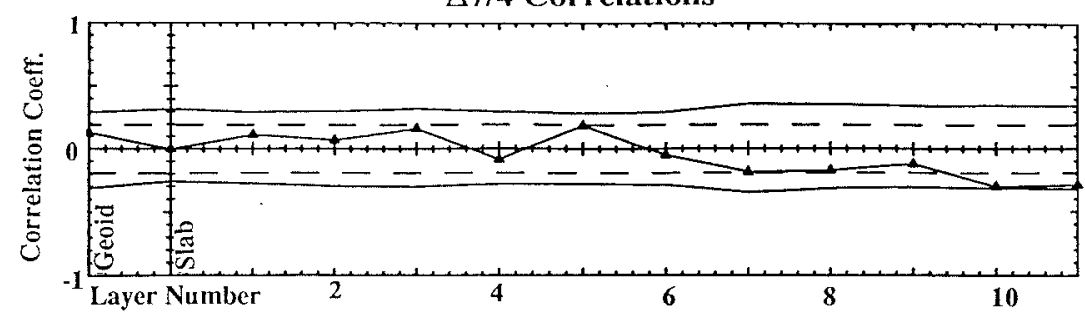

$\Delta 8 / 4$ Correlations

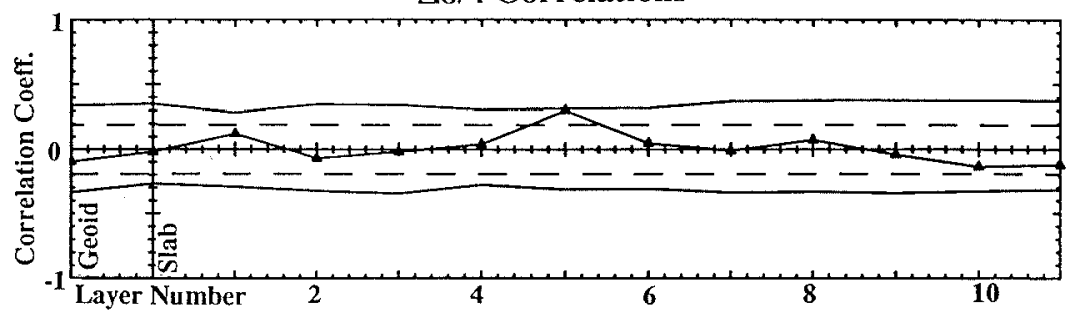

$\triangle \mathrm{Sr}$ Correlations

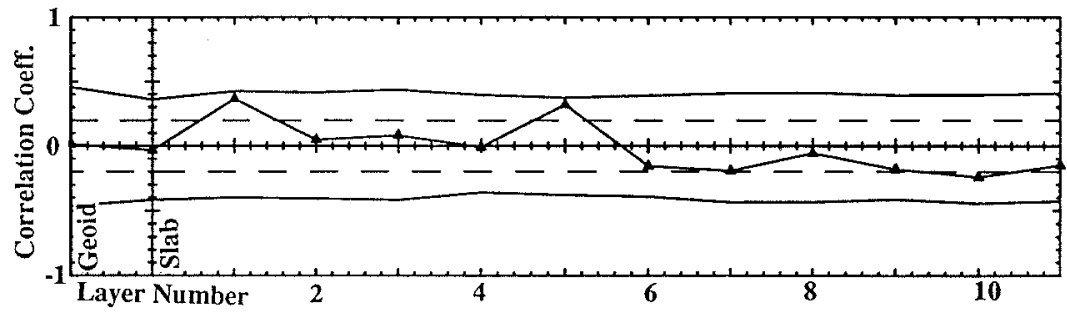

$\Delta \mathrm{Nd}$ Correlations

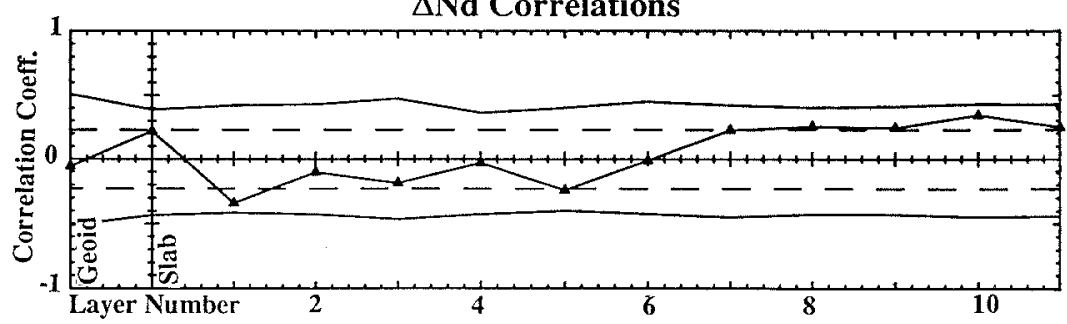

Figure 3. Plots of the spatial correlation between geochemical isotopic data and the geoid, subducted slab, and Tanimoto [1990] tomography model. The solid line with solid triangles is the calculated correlation coefficients. The dotted lines are the 95\% confidence level lines for the standard linear correlation test, and the solid lines with no symbols are the $95 \%$ confidence level lines for the Monte Carlo style significance test discussed in the text. The horizontal axis is the shell number of the tomography (1-11), with geoid and subducted slab as labeled. The vertical axis is the correlation coefficient.

hotspots with ridges. Using $5^{\circ} \times 5^{\circ}$ bins, we find 15 bins contain both ridges and hotspots, 99 bins contain hotspots and no ridges, 201 bins contain ridges and no hotspots, and 2135 bins contain neither hotspots nor ridges. Clearly, this results in a large positive correlation coefficient which does not tell us that hotspots tend to be located in the same bins as ridges are. This correlation tells us that bins which contain no hotspots typically contain no ridges, which is not the same thing at all. As with any experiment, the correlation must be designed to answer the right question.

The correlation of both lists of hotspot locations with the geoid, post-Pangeatic subduction, and the Tanimoto [1990] tomography model is shown in Figure 4 . The most striking correlations are the positive correlation of both sets of hotspot locations with the geoid, which exceeds both the $95 \%$ confidence level line for the binomial distribution test and the
95\% confidence level line from the Monte Carlo test. Recalling Table 1, we remember that the spherical harmonic coefficient correlations showed no statistically significant correlation between geoid and the short list of hotspots, but the spatial correlation immediately reveals it. Beyond this correlation, however, there are no correlations which are statistically significant under the Monte Carlo test. The correlations between slow regions in layers 7-11 (1555-2891 km) and the short list of hotspot locations are strong but still below the $95 \%$ confidence level.

From this figure, we must conclude that the hotspot locations do not correlate significantly with slow regions of the lowermost mantle, at least as compared to randomly oriented sets of hotspots with the relative positions of the hotspots fixed to each other. It is important to recall that the correlation technique being used here does not consider the magnitude of the data in 

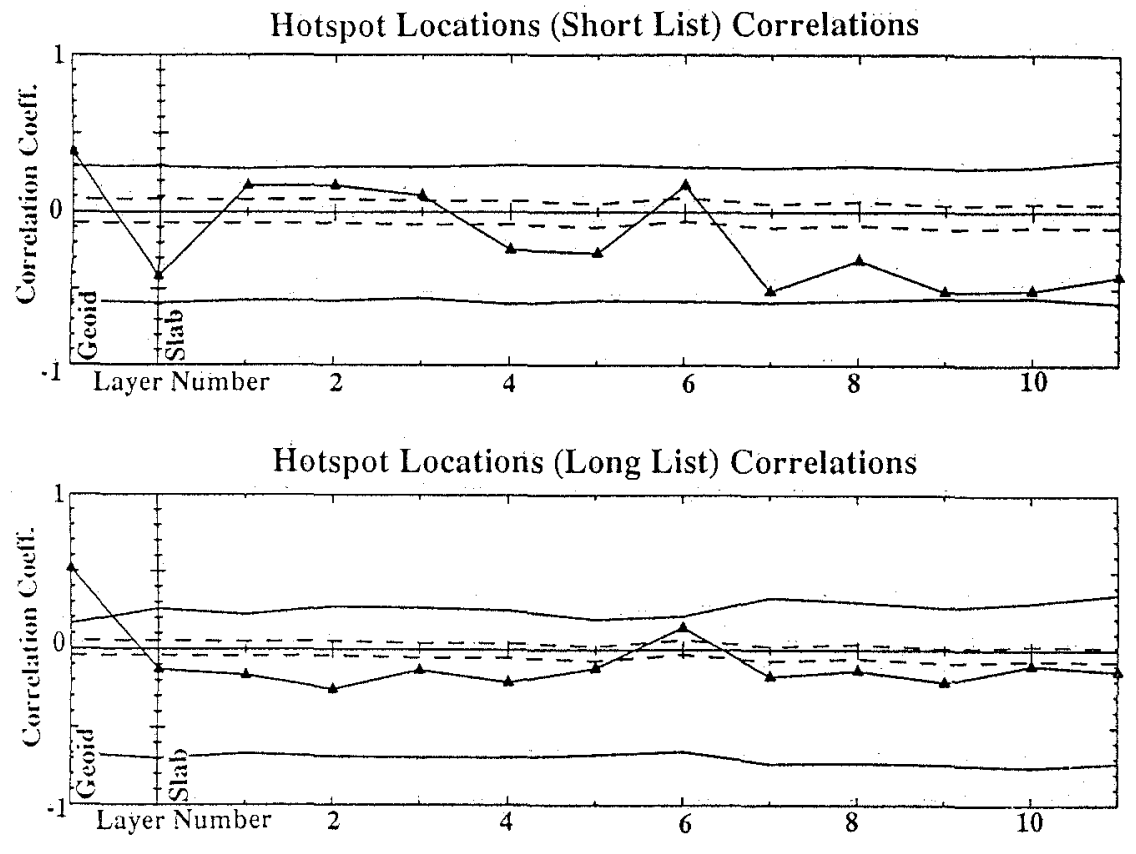

Figure 4. Plots of the spatial correlation between both lists of hotspot locations and the geoid, subducted slab, and Tanimoto [1990] tomography model. Plotting symbols and axes are identical to those in Figure 3.

the tomography, geoid, or slab data sets; the test only considers the sign of the points. If the magnitudes at the points were to be included, these correlations might be stronger. The fact that correlations between the geoid and hotspot locations is quite strong in the spatial domain but nonexistent with the low-degree spherical harmonic expansions is probably explained by the smoothing action of the low-order spherical harmonic expansion of hotspot locations.

The correlations for the ridge locations and subducted slab are shown in Figure 5. (The plots in Figure 5 have the same format as Figure 4.) The only statistically significant correlation for ridge locations is in layer $6(1284-1555 \mathrm{~km})$ between the location of ridge and fast regions of the mantle. Recall that the strongest correlation in the spherical harmonic expansion of ridges was for layer 6 at degree 4 . The next strongest correlation is between the location of ridges and slow regions of the uppermost mantle $(0-220 \mathrm{~km})$, but this correlation is not quite significant at the $95 \%$ level. Ridges also correlate with the lowermost mantle in layers 8-11 $(1816-2891 \mathrm{~km})$ nearly as strongly as the short list of hotspots. Accepting the correlations

Oceanic Ridge Locations Correlations

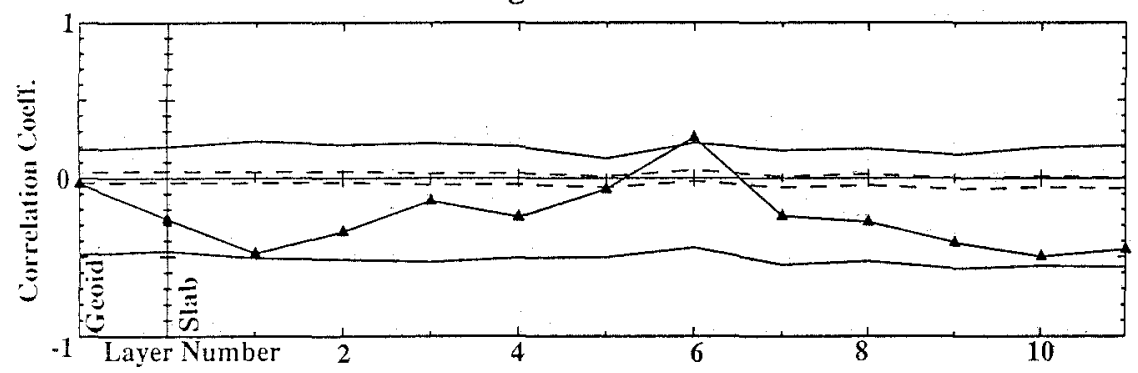

Subducted Slab Locations Correlations

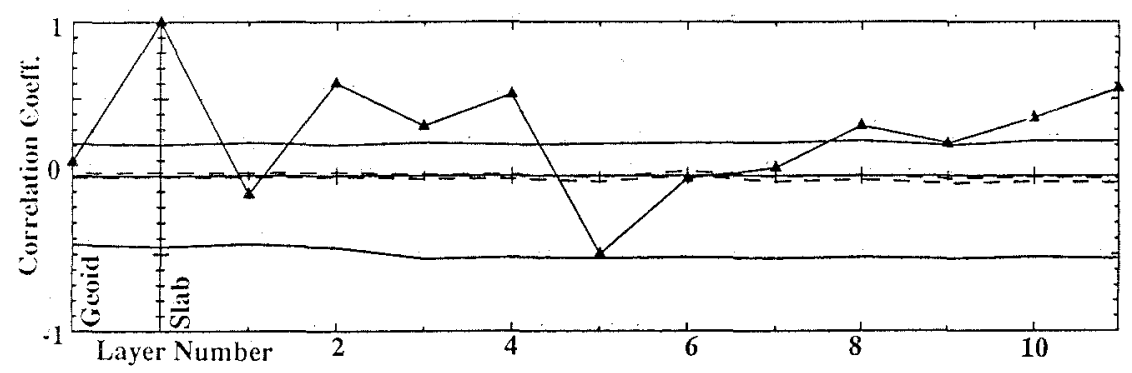

Figure 5. Plots of the spatial correlation between oceanic ridge locations and subducted slab and the geoid, subducted slab, and Tanimoto [1990] tomography model. Plotting symbols and axes are identical to those in Figure 4. 
in the upper part of Figure 4 (or those in Figure 3) in the lowermost mantle as statistically significant would seem to require accepting the deep mantle correlations for the ridge locations as statistically significant, implying that ridges have their origin in the lowermost mantle, which does not at all seem reasonable.

Tuming to the locations of post-Pangean subducted slab, we find the correlations shown in the lower part of Figure 5. Disregarding the perfect self-correlation, we see numerous statistically significant correlations between the locations of slab and fast regions of the middle mantle $(220-1022 \mathrm{~km})$ and lowermost mantle $(1816-2891 \mathrm{~km})$ as well as between slab and slow regions of layer $5(1022-1284 \mathrm{~km})$. The middle mantle correlations agree with those of Scrivner and Anderson [1992], but they found no correlations in the lowermost mantle (for this tomographic model). The correlations in the lowermost mantle may correspond to those found by Richards and Engebretson [1992], but, in contrast to their interpretation, only a fraction of the lower mantle is involved. The middle lower mantle exhibits no correlation. Significantly, the spatial-domain correlations show both the middle mantle and lowermost mantle correlations, while attempts with spherical harmonics have only found one or the other.

\section{Discussion}

The slab correlations in Figure 5 demand some form of coupling or communication between the upper and lower mantle. If the slabs penetrate through the $670 \mathrm{~km}$ discontinuity, which is the interpretation of Richards and Engebretson [1992], the slabs would experience a phase change as they do so and the result would be a strong signal in the transition zone where this phase change is occurring. In the middle lower mantle we could lose this signal since the slab would be a rather narrow feature and perhaps not have a sufficiently strong variation in velocity to be seen at the resolution of the tomography model. The signal might reappear as the slab crumples and spreads out near the core-mantle boundary. Alternatively, the slabs may fail to penetrate the $670-\mathrm{km}$ discontinuity, as argued by Scrivner and Anderson [1992] and instead, simply spread out at the base of the upper mantle. These might then induce cold downwellings (thermal coupling or topographic coupling) to form beneath them which are rather narrow in the middle lower mantle and spread out near the core-mantle boundary. A third and perhaps more inviting alternative is the "avalanche" downwelling seen by Tackley et al. [1993] in their three-dimensional convection model with internal heating and a phase change at the $670-\mathrm{km}$ discontinuity. In this scheme, the slabs would be stopped temporarily at the transition zone and spread out laterally. After some time, in a chemically and rheologically uniform mantle, a catastrophic downwelling (avalanche) occurs, and slab material, along with other middle mantle material cooled by the slab, falls relatively rapidly to the core-mantle boundary where it then spreads out. This model is tempting because it implies that the downwelling material is transitory in the middle lower mantle. The correlation between slab and slow regions from 1284-1555 $\mathrm{km}$ depth suggests that the slabs do stop at the $670-\mathrm{km}$ discontinuity. This correlation argues against a long-lived stable downwelling beneath the slab and seems to support the "avalanche" type of downwelling. The slab correlations in Figure 5 are also striking in that these are the strongest correlations found in the lower mantle using the spatial domain correlation techniques. This fact strongly suggests that slabs and associated downwellings are the dominant factor in mantle convection.

An alternative model is the following: The breakup of a supercontinent causes slab cooling in the mantle beneath the leading edges of the moving continents. Continents eventually come to rest over cold downwellings and resist overriding hot upwelling mantle regions. Mantle affected by subduction is essentially cooled (or not heated) from below (and above, for the lower mantle, if slabs do not penetrate). Subsequent subduction and continent standstills tend to occur over mantle previously cooled. Subduction tends to repeat in the same general area so one expects a correlation between integrated slab positions and tomography even if cold regions of the mantle are not directly caused by the most recent supercontinent cycle [Anderson, 1994].

One feature which is quite obvious in Figures 4, 5, and 6 is the asymmetry of the Monte Carlo confidence level lines. This does not appear to be due to the algorithms for randomly reorienting the data spheres or determining the confidence level lines since the same algorithms were used to find the symmetrically arranged confidence level lines of Figure 3 . This may be due to an inherent skewness in the test, but the Monte Carlo significance testing is designed take this into account. Since the test depends on the percentage of positive and negative area on the map, we expect a skewness if the map does not have equal positive and negative areas. The binomial distribution test assumes that the locations of the points being "thrown" at the map have no dependence on the locations of other points (which is certainly not true of the ridge and slab locations). Another complicating factor is that each of the $5^{\circ} \times$ $5^{\circ}$ bins may only be occupied once, so the proportions of positive and negative areas available for each successive point to land on are altered by the placement of previous points. In hindsight, the use of the binomial test as a test of significance was a poor choice, and its results should be ignored in favor of the Monte-Carlo test.

\section{Putting it Together}

Figure 6 shows the spatial domain correlation between the locations of the OIB geochemistry data points (a subset of the hotspot list) and geoid, slab, and mantle tomography. This shows that the location of these data points correlate very well with slow regions of the lowermost mantle (1555-2630 km) and with high regions of the geoid. This suggests that strong correlations between the data values and these regions of the mantle should be viewed with suspicion. The typical null hypothesis used as the basis for tests of statistical significance is the hypothesis that the functions being correlated with each other are actually uncorrelated. Note that the correlations of Figure 6, which are for a biased subset of the hotspot locations, are much better than for either the short or the long hotspot locations list.

Taking a case of isotopic geochemistry data collected from hotspot locations as an example, a correlation is found between positive deviations from the reference line and slow regions in the lower mantle (e.g., 1816-2088 km depth). However, it is known that isotopic ratios from samples collected at hotspot locations always show positive deviations from the reference line for this particular set of isotopes (possibly by definition, i.e., positive deviation is a hotspot and negative or no deviation is not a hotspot). When a correlation is performed between the hotspot locations and the mantle tomography, a strong 


\section{Data Point Locations Correlation}

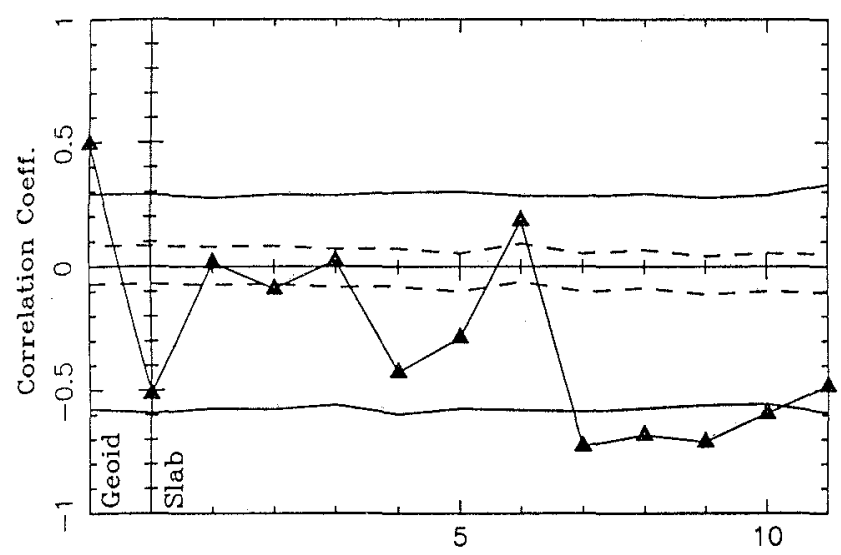

Layer Number

Figure 6. Plot of the spatial correlation between ocean island basalt data point locations (open circles in figure 2) with the geoid, subducted slab, and tomography. Plotting symbols, axes and sense of correlation are the same as in Figure 4.

correlation is found between the hotspot locations and slow regions in the lower mantle (1816-2988 $\mathrm{km}$ depth). In this case, the correlation of the isotopic ratios with the mantle tomography is trivial.

An alternative case is a case of a very low correlation coefficient resulting from the correlation of geochemical data collected at hotspot locations with tomography (e.g., 1816-2088 $\mathrm{km}$ depth). Again the hotspot locations correlate strongly with slow regions of the lower mantle. Additionally, no particular relationship between hotspot locations and the isotopic ratios is known to exist. Again the (lack of) correlation of the isotopic ratios and tomography is rather trivial, simply stating that the isotopic ratios and the hotspot locations are unrelated, which was known anyway.

In both of the preceding cases, if the relationship between the geochemistry and the hotspot location was unknown to begin with, the correlation between the geochemistry and the mantle tomography is significant in revealing the relationship between the geochemistry and the hotspot locations. However, in neither case is anything learned from the correlation between geochemistry and tomography which could not have been determined through the much simpler means of checking for a relationship between the geochemical isotopic ratios and samples collected at hotspot locations and samples collected at non-hotspot locations. Statistical techniques for determining whether one correlation coefficient is significantly better than another do exist [Press et al., 1986], but there are very strict requirements on the distribution of the functions being correlated. The fundamental point is that an attempt must be made to assess the relationship between the data collection locations and the function that the data is to be correlated with (e.g., tomography, the geoid, and subducted slab) before any assessment of the meaningfulness of the correlation between the it data and the function can be made.

Column 5 of Table 1 shows the significant spherical harmonic correlation of the neodymium data points with the slab, geoid, and tomography. Compare this with the correlation between the neodymium isotopic data and the tomography, slab, and geoid in Column 4 of Table 1. Clearly, the pattern of correlations for the data point locations is quite similar to that for the data values. This suggests that the neodymium spherical harmonic data correlations are much less significant than they appear in Table 1.

We have seen that an examination of the correlations of the data point locations with the tomography, slab, and geoid casts doubt on many of the correlations claimed for the isotopic data. It may be possible to extract the effect of the locations through extensive statistical testing. The Monte Carlo technique used in this paper to estimate significance levels certainly diminishes the significance of all of the isotopic correlations to below $95 \%$. This casts doubt on previous claims that hotspot geochemistry originates in the lower mantle.

\section{Conclusions}

It is clear that the use of spherical harmonic coefficients to comelate geochemical data with geodynamic data is inappropriate because of the irregular and sparse sampling. In the case of such fields as the global hotspot, ridge, and slab distributions, we know (in principle or by assumption) the field at every point on the surface and can therefore avoid the orthogonality problems arising when this is not the case. Once again, we cannot use a spherical harmonic expansion to demonstrate that these fields have the property of arising from physical processes which result in spherical harmonic solutions.

The spherical harmonic correlations of the isotopic data give results that seem to conflict internally or that seem to arise from the distribution of the data points on the sphere. When the correlations are performed in the spatial domain, the correlations are more consistent internally, and the distribution of the data points on the sphere is revealed to be an important

factor in these correlations. The effect of the distribution of the data on the surface given the form of the geodynamic field can be answered partially through a Monte Carlo test by asking, What is the probability of a correlation coefficient at least as large as this resulting from a random reorientation of the tomography, geoid, or slab shell? The results of this test indicate that the correlations are actually not statistically significant at all.

Turning to the distributions of hotspots, ridge, and slab; we find that the spherical harmonic correlations show very strong and statistically significant correlations between the short list of hotspots and slow regions of the lowermost mantle as well as between the slab locations and fast regions of the transition zone. These comelations also occur in spherical harmonic degrees with high power in both of the expansions being correlated. In the case of both ridge and the long list of hotspots the statistically significant correlations which do occur tend to be in low power degrees of at least one of the two distributions being correlated. Notably lacking is a correlation between the spherical harmonic terms of the hotspot distributions and the geoid, which has been apparently well documented in the past [Richards and Engebretson, 1992], although the correlations found were from a slab-removed geoid model and, thus, model dependent. The geoid model in this paper still contains any slab signature that may be present.

Spatial domain correlations of both hotspot lists reveal a very strong and statistically significant correlation between high regions of the geoid and the hotspot distribution even with the non-slab-removed geoid model. However, neither list of hotspots shows statistically significant correlations with lowermost mantle tomography. Ridge locations show a 
statistically significant correlation with fast regions of the middle lower mantle (1284-1555 km), and correlations nearly as strong as those of the short list of hotspots in the lowermost mantle. If we would like to accept the correlations of the geochemistry and hotspot locations which fall just short of the 95\% confidence level lines to argue for a deep mantle signature in those fields, we must then accept the equally strong correlations of the ridge locations and therefore argue that they too have a deep mantle signature which is in conflict with the generally held view that ridges are passive and shallow features.

The most striking and potentially meaningful correlations are those between the integrated slab map (the "slab shell") and fast regions of both the middle mantle $(220-1022 \mathrm{~km})$ and the lowermost mantle $(1816-2891 \mathrm{~km})$. These correlations are very strong, statistically significant, internally consistent, and extensive in the sense that the correlations are not just in one or two isolated layers. These correlations support a model where slab is temporarily stopped at the $670 \mathrm{~km}$ discontinuity, builds up, and occasionally "avalanches" to the core-mantle boundary. This is similar to the model results of Tackley et al. [1993]. Alternatively, cold downwelling regions of the mantle tend to persist and these control continent and subduction zone locations. The cold parts of the lowermost mantle may be left over from a previous subduction episode. The strength of these correlations as compared to those of all of the other fields considered indicates that slabs, and perhaps internal mantle downwellings induced by slabs, are the dominant factor in determining the form of mantle convection. Upwellings may therefore be restricted to other regions or may, in fact, be mantle displaced by downwellings rather than thermal boundary layer instabilities. Long wavelength anomalies in the mantle may be due to supercontinents [Anderson, 1982; Gurnis, 1988], slab cooling [Scrivner and Anderson, 1992], or temperature dependent viscosity (P. J. Tackley, personal communication, 1992).

Acknowledgments. We thank Craig Scrivner for digital maps of integrated subduction, Sharon Kedar for digital listings of hotspot locations, Thomas Kiefer for the digital geoid model, and Toshiro Tanimoto for the digital tomography model. This work was supported by NSF grant EAR 90-02947 and a NASA Graduate Fellowship. Contribution 5302, Division of Geological and Planetary Sciences, Caltech, Pasadena, CA 91125.

\section{References}

Anderson, D. L., Superplumes or supercontinents?, Geology, 22, 3942, 1994.

Anderson, D. L., Hotspots, polar wander, mesozoic convection, and the geoid, Nature, 297, 391-393, 1982.

Cain, J. C., W. E. Daniels, S. J. Hendricks, and D. C. Jensen, An evaluation of the main geomagnetic field, 1940-1962, J. Geophys. Res., 68, 1131-1139, 1965.

Castillo, P., The Dupal anomaly as a trace of the upwelling lower mantle, Nature, 336, 667-670, 1988.

Crough, S. T., and D. M. Jurdy, Subducted lithosphere, hotspots and the geoid, Earth Planet. Sci. Lett., 48, 15-22, 1980.

Eckhardt, D. H., Correlations between global features of terrestrial fields, Math. Geol., 16, 155-171, 1984.

Foguere, P. F., Spherical harmonic analysis, 2, A new model derived from magnetic observatory data for epoch 1960.0, J. Geophys. Res., 70, 2171-2179, 1965.

Gaposchkin, E. M., Earth's gravity fields to the eighteenth degree and geocentric coordinates for 104 stations from satellite and terrestrial data, J. Geopys. Res., 79, 5377-5411, 1974.

Gill, J. B., Sr-Pb-Nd isotopic evidence that both MORB and OIB sources contribute to oceanic island arc magmas in Fiji, Earth Planet. Sci. Lett., 68, 443-458, 1984.

Gurnis, M., Large-scale mantle convection and the aggregation and dispersal of supercontinents, Nature, 332, 695-699, 1988.

Hart, S. R., A large-scale isotope anomaly in the Southern Hemisphere mantle, Nature, 309, 753-787, 1984.

Hurwitz, L., D. G. Knapp, J. H. Nelson, and D. E. Watson, Mathematical model of the geomagnetic field for 1965, $J$. Geophys. Res., 71, 2373-2383, 1966.

Inoue, H., Y. Fukao, K. Tanabe, and Y. Ogata, Whole mantle P-wave travel time tomography, Phys. Earth Plan. Int., 59, 294-328, 1990.

Ito, E., W. M. White, and C. Gopel, The $\mathrm{O}, \mathrm{Sr}, \mathrm{Nd}$, and $\mathrm{Pb}$ isotope geochemistry of MORB, Chem. Geol., 62, 157-176, 1987.

Kedar, S., D. L. Anderson, and D. J. Stevenson, Relationship between hotspots and mantle structure: Correlation with whole mantle seismic tomography, in Proceedings of the NATO Advanced Study Institute on Dynamic Modeling and Flow in the Earth and Planets, in press, 1994.

Leaton, B. R., S. R. C. Malin, and M. J. Evans, An analytic representation of the estimated geomagnetic field and its secular change for epoch 1965.0, J. Geomagn. Electr., 17, 187-194, 1965.

Morgan, W. J., Hotspot tracks and the opening of the Atlantic and Indian oceans, in The Sea, edited by C. Emiliani, pp. 443-487, Wiley-Interscience, New York, 1981.

Press, W. H., B. P. Flannery, S. A. Teukolsky and W. T. Vetterling, Numerical Recipes: The Art of Scientific Computing, 818 pp., Cambridge University Press, New York, 1986.

Richards, M. A., and D. C. Engebretson, Large scale mantle convection and the history of subduction, Nature, 355, 437-440, 1992.

Richards, M. A., B. H. Hager, and N. H. Sleep, Dynamically supported geoid highs over hotspots: Observation and theory, $J$. Geophys. Res., 93, 7690-7708, 1988.

Richardson, S. H., A. J. Erlank, A. R. Duncan, and D. L. Reid, Correlated $\mathrm{Nd}, \mathrm{Sr}$ and $\mathrm{Pb}$ isotope variation in Walvis Ridge basalts and implications for the evolution of the mantle source, Earth Planet. Sci. Lett., 59, 327-342, 1982.

Roden, M. K., S. R. Hart, F. A. Frey, and W. G. Meison, Sr, Nd and $\mathrm{Pb}$ isotopic and REE geochemistry of St. Paul's Rock: The metamorphic and metasomatic development of an alkali basalt mantle source, Contrib. Mineral. Petrol., 85, 376-390, 1984.

Scrivner, C. and D. L. Anderson, The effect of post-Pangea subduction on global mantle tomography and convection, Geophys. Res. Lett., 19, 1053-1056, 1992.

Su, W., and A. M. Driewonski, Predominance of long-wavelength heterogeneity in the mantle, Nature, 352, 121-126, 1991.

Tackley, P. J., D. J. Stevenson, G. A. Glatzmaier, and G. Schubert, Effects of an endothermic phase-transition at $670 \mathrm{~km}$ depth in a spherical model of convection in the Earth's mantle, Nature, 361, 699-704, 1993.

Tanimoto, T., Long Wavelength S-wave velocity structure throughout the mantle, Geophys. J. Int., 100, 327-336, 1990

Vidal, P., E. Chauvel, and R. Brousse, Large mantle heterogeneity beneath French Polynesia, Nature, 307, 536-538, 1984.

Vogt, P. R., On the applicability of thermal conduction models to midplate volcanism: Comments on a paper by Gass et al., J. Geophys. Res., 86, 950-960, 1981.

Zindler, A., E. Jagoutz, and S. Goldstein, Nd, Sr, and Pb isotopic systematics in a three-component mantle: a new perspective., Nature, 298, 519-523, 1982.

D. L. Anderson and T. W. Ray, Seismological Laboratory, Califomia Institute of Technology, 170-25, Pasadena, CA 91125 (e-mail: dla@earth.gps.caltech.edu; terrill@mars1.gps.caltech.edu)

(Received July 19, 1993; revised January 28, 1994;

accepted January 31, 1994.) 